\title{
Evidence that SNF2/SWI2 and SNF5 activate transcription in yeast by altering chromatin structure
}

\author{
Joel N. Hirschhorn, Steven A. Brown, ${ }^{1}$ Chris D. Clark, ${ }^{2}$ and Fred Winston ${ }^{3}$ \\ Department of Genetics, Harvard Medical School, Boston, Massachusetts 02115 USA
}

\begin{abstract}
Changes in chromatin structure have frequently been correlated with changes in transcription. However, the cause-and-effect relationship between chromatin structure and transcription has been hard to determine. In addition, identifying the proteins that regulate chromatin structure has been difficult. Recent evidence suggests that a functionally related set of yeast transcriptional activators (SNF2/SWI2, SNF5, SNF6, SWI1, and SWI3), required for transcription of a diverse set of genes, may affect chromatin structure. We now present genetic and molecular evidence that at least two of these transcriptional activators, SNF2/SWI2 and SNF5, function by antagonizing repression mediated by nucleosomes. First, the transcriptional defects in strains lacking these $S N F$ genes are suppressed by a deletion of one of the two sets of genes encoding histones $\mathrm{H} 2 \mathrm{~A}$ and H2B, (hta1-htb1) $\Delta$. Second, at one affected promoter (SUC2), chromatin structure is altered in snf2/swi2 and snf5 mutants, and this chromatin defect is suppressed by (hta1-htb1) 4 . Finally, analysis of chromatin structure at a mutant SUC2 promoter, in which the TATA box has been destroyed, demonstrates that the differences in SUC2 chromatin structure between $S N F 5^{+}$and snf5 mutant strains are not simply an effect of different levels of $S U C 2$ transcription. Thus, these results strongly suggest that SNF2/SWI2 and SNF5 cause changes in chromatin structure and that these changes allow transcriptional activation.
\end{abstract}

[Key Words: Yeast; chromatin; transcriptional activation; SNF and SWI proteins]

Received August 10, 1992; revised version accepted September 17, 1992.

Understanding the mechanisms by which genes are transcriptionally activated and repressed is central to understanding gene regulation. Numerous studies have shown a correlation between changes in transcription and alterations in chromatin structure (for review, see van Holde 1988; Grunstein 1990a). These studies have suggested that the positions of nucleosomes, the primary components of chromatin, may affect transcription. However, the cause-and-effect relationship between changes in transcription and alterations in chromatin structure is unclear.

Recent genetic and biochemical data strongly support the view that nucleosomes play an important role in transcriptional regulation. Studies in the yeast Saccharomyces cerevisiae have provided genetic evidence that histones, the protein components of nucleosomes, affect transcription. For example, alterations in histone stoichiometry restore transcription to promoters disrupted by certain transposon insertion mutations (Clark-Adams et al. 1988). In addition, depletion of histone $\mathrm{H} 4$ or mutations in genes encoding histone $\mathrm{H} 4$ can result in in-

Present addresses: ${ }^{1}$ Department of Molecular Biology, Massachusetts General Hospital, Boston, Massachusetts 02114 USA; ${ }^{2}$ Department of Genetics, Stanford University, Stanford, California 94305 USA.

${ }^{3}$ Corresponding author. creased or decreased levels of transcription (for review, see Grunstein 1990b). Genetic and biochemical data also suggest that some transcriptional activators may function to alleviate the repressive effects of chromatin structure (Fascher et al. 1990; Croston et al. 1991; Workman et al. 1991). However, aside from histones, the proteins involved in the regulation of chromatin structure are largely unknown.

A series of genetic experiments led us to believe that three yeast transcriptional activators, SNF2/SWI2 (hereafter referred to as SNF2 for simplicityl, SNF5, and SNF6, might function by altering chromatin structure. The SNF genes were originally identified by mutations causing defects in transcription of SUC2, a glucose-repressible gene that encodes the enzyme invertase (Carlson et al. 1981; Neigeborn and Carlson 1984). SNF2, SNF5, and SNF6 were also shown to be required for the expression of a number of diversely regulated genes (Abrams et al. 1986; Estruch and Carlson 1990; Happel et al. 1991) and thus appear to encode transcriptional activators.

Two genetic studies provided the link between SNF proteins and chromatin structure (for review, see Winston and Carlson 1992). The first study, in which suppressors of snf2 and snf5 mutations were isolated, identified the gene SPT6 (Neigeborn et al. 1986). A separate study, in which suppressors of transposon insertion mu- 
tations were isolated, identified not only SPT6 but also the gene pair HTA1-HTB1, one of two sets of genes encoding the histones $\mathrm{H} 2 \mathrm{~A}$ and $\mathrm{H} 2 \mathrm{~B}$ (Winston et al. 1984; Clark-Adams et al. 1988; Fassler and Winston 1988). Furthermore, mutations in SPT6 and in HTA1-HTB1 were shown to cause many similar phenotypes (Clark-Adams et al. 1988; Fassler and Winston 1988). Because spt6 mutations suppress snf2, snf5, and snf6 mutations and because spt6 mutations cause many of the same phenotypes as do $h t a 1-h t b 1$ mutations, it seemed possible that the histone mutations might also suppress snf2, snf5, and snf6. This suppression, presented in this paper, indicates that the function of SNF2, SNF5, and SNF6 transcriptional activators might involve chromatin.

More recent genetic studies have shown that SNF2, SNF5, and SNF6 are part of a larger group of functionally related proteins. This set of SNF proteins overlaps with a set of proteins named SWI1, SWI2, and SWI3 (Stern et al. 1984). Mutations in SWI genes, isolated on the basis of defects in transcription of the $H O$ gene, also affect transcription of a diversely regulated set of genes (Stern et al. 1984; Peterson and Herskowitz 1992). Recent work showed that SWI2 is identical to SNF2 (Laurent et al. 1991; K. Nasmyth, pers. comm.). In every case tested, mutations in snf2, snf5, snf6, swi1, and swi3 cause defects in transcription of a diverse set of genes, including SUC2, Ty elements, INO1, GAL1, GAL10, and $H O$ (for review, see Winston and Carlson 1992). Interestingly, suppressors of swi1 mutations have identified a gene (SIN1/SPT2) that shows similarity to HMG proteins (nonhistone components of chromatin), further strengthening the idea that the function of the SNF and SWI activators is related to chromatin (Kruger and Herskowitz 1991).

The SNF and SWI proteins have been proposed to participate in a common function, possibly as a heteromeric complex (Laurent et al. 1991; Peterson and Herskowitz 1992). This function may require helicase activity because SNF2 has homology to known helicases (Davis et al. 1992; Laurent et al. 1992). In addition, although the SNF and SWI proteins have not been shown to bind to DNA, at least SNF2, SNF5, and SNF6 appear to play a direct role in transcriptional activation because lexASNF protein fusions can activate transcription by a $\operatorname{lex} A$ operator (Laurent et al. 1990, 1991; Laurent and Carlson 1992).

In this paper we provide evidence that at least two of these proteins, SNF2 and SNF5, function by altering chromatin structure. First, we show that (hta1-htb1) $\Delta$ suppresses multiple transcriptional defects of snf2 and snf5 mutants, including a defect in SUC2 transcription. We then show that the chromatin from snf2 and snf5 mutants exhibits a different pattern of cleavage by micrococcal nuclease (MNase) over the SUC2 promoter region than does chromatin from $\mathrm{SNF}^{+}$strains. In addition, the suppression of snf2 and snf5 by $(h t a 1-h t b 1) \Delta$ is reflected in the MNase cleavage patterns over this region. Finally, by examining strains carrying mutations in the SUC2 TATA box, we show that the differences in chromatin structure are not caused by different levels of transcription. Taken together, these results indicate that SNF2, SNF5, and the other SNF and SWI proteins activate transcription by altering chromatin structure. Because SNF2 has sequence homologs in many other organisms (Tamkun et al. 1992; other references are cited in Laurent et al. 1992), this mechanism of activation may be functionally conserved throughout eukaryotes.

\section{Results}

Decreased histone gene dosage suppresses mutations in certain SNF genes

Yeast strains carrying mutations in SNF2, SNF5, or SNF6 are defective for transcription of a diversely regulated set of genes, including SUC2, Ty elements, INO1, GAL1, and GAL10 (Neigeborn and Carlson 1984; Happel et al. 1991; Peterson et al. 1991; Peterson and Herskowitz 1992). Preliminary evidence indicated that (hta1-htb1) $\Delta$, a deletion of one of two gene pairs that encode histones $\mathrm{H} 2 \mathrm{~A}$ and $\mathrm{H} 2 \mathrm{~B}$, might suppress snf2, snf5, and snf6 mutations for the defects caused in SUC2 expression /ClarkAdams 1988). This deletion is believed to reduce the levels of histones H2A and H2B in vivo (Clark-Adams et al. 1988).

To examine more rigorously the suppression of snf mutations by (hta1-htb1) $\Delta$, we constructed a set of isogenic strains, differing only with respect to their $S N F$ and HTA1-HTB1 genotypes. We then measured the levels of invertase, the SUC2 gene product, in these strains. Because SUC2 is transcriptionally regulated, measurement of invertase levels reflects the level of SUC2 mRNA (Carlson 1987). Our results (Table 1) show that the SUC2 transcriptional defects of snf2, snf5, and snf6 mutants

Table 1. The effects of snf, hta1-htbl, and suc2 mutations on SUC2 gene expression

\begin{tabular}{lcc}
\hline & \multicolumn{2}{c}{ Invertase activity $^{\mathrm{b}}$} \\
\cline { 2 - 3 } Relevant genotype $^{\mathrm{a}}$ & HTA1-HTB1 & $($ hta1-htb1) \\
\hline SNF $^{+}$SUC2 $^{+}$ & 100 & 98 \\
snf2 & 13 & 38 \\
snf5 & 7 & 60 \\
snf6 & 12 & 37 \\
snf1 & $<1$ & $<1$ \\
snf4 & $<1$ & $<1$ \\
suc2-104 & 5 & 4 \\
snf5 suc2-104 & $<1$ & 1
\end{tabular}

${ }^{a}$ Relevant genotype indicates the SUC2 and SNF genotypes. The two columns of data show the secreted invertase activities for strains that are $H T A 1-H T B 1^{+}$or $(h t a 1-h t b 1) \Delta$, respectively, in conjunction with the indicated $S U C 2$ and SNF genotypes. The strains used for these assays include all of the strains listed in Table 2 with the exception of FY637. In some cases, the invertase values represent the average from more than one strain.

bUnits shown are micromoles of glucose released per minute per $100 \mathrm{mg}$ of dry weight cells. Values shown are the average of at least two different derepressed cultures; two different amounts of cells were assayed for each culture. All repressed cultures had $<1$ unit of activity. All standard errors were $<15 \%$. 
are suppressed by (hta1-htb1) $\Delta$ : Strains carrying (hta1$h t b 1 \mid \Delta$ in combination with a snf2, snf5, or snf6 mutation express invertase at a level three- to ninefold above that observed in strains carrying only the $s n f$ mutations. Northern hybridization analysis confirms that SUC2 mRNA levels in wild-type, (hta1-htb1) $\Delta$, snf5, and snf5 (hta1-htb1) $\Delta$ strains correlate with the levels of invertase activity measured for these strains (data not shown). This suppression suggests a functional interaction between SNF proteins and histones.

Suppression by the histone mutation $(h t a 1-h t b 1 \mid \Delta$ is specific to snf2, snf5, and snf6 mutations in all cases tested; that is, suppression does not occur by generally increasing SUC2 transcription. First, (hta1-htb1) $\Delta$ does not detectably affect normal SUC2 expression in a $S N F^{+}$ background, under either repressing (high glucose) or derepressing (low glucose) conditions (Table 1). In addition, (hta1-htb1) $\Delta$ does not suppress a mutation in the SUC2 TATA box (suc2-104; Table 1). Finally, (hta1$h t b 1) \Delta$ has no detectable effect on $\operatorname{snf1}$ or $\operatorname{snf} 4$ mutations (Table 1); snf1 and snf4 mutations are believed to impair $S U C 2$ transcription by a different mechanism than snf2, snf5, and snf6 mutations (Neigeborn et al. 1986). Thus, suppression of $\operatorname{snf2}$, snf5, and $\operatorname{snf6}$ by $(h t a 1-h t b 1) \Delta$ is unlikely to be the result of a nonspecific increase in transcription at SUC2. More probably, this histone deletion suppresses a specific transcriptional defect caused by snf2, snf5, and snf6 mutations.

Previous work showed that snf2, snf5, and snf6 strains, but not $s n f 1$ or snf 4 strains, were defective for transcription of the yeast transposable elements Ty1 and Ty2 (Ciriacy et al. 1991; Happel et al. 1991). Therefore, we also determined whether deletion of the HTA1-HTB1 locus suppresses the defect of $s n f 2, \operatorname{snf5}$, and snf6 strains for transcription of these Ty elements. Northern hybridization analysis (Fig. 1) demonstrates that (hta1-htb1) $\Delta$ reproducibly suppresses the Ty transcription defect of snf2 and snf5 mutants. The effect of $(h t a 1-h t b 1) \Delta$ on the snf6 defect is less clear (Fig. 1, lanes 7, 8). In the experiment shown, (hta1-htb1) $\Delta$ does not appear to suppress the Ty transcription defect of snf6. In other experiments weak suppression has been observed (data not shown).

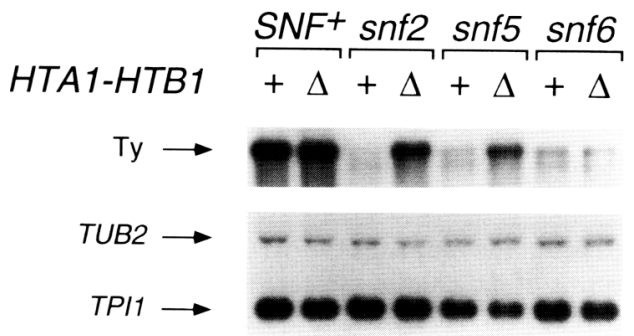

Figure 1. A deletion of HTA1-HTB1 suppresses the Ty transcription defect in snf2 and snf5 mutant strains. Total RNA was prepared from each strain and subjected to Northern analysis. Ten micrograms of RNA was run in each lane. The filter was hybridized with a Ty probe and rehybridized with TUB2 and TPI1 probes for standardization. The strains were (from left to right): FY120, FY710, FY458, FY724, FY712, FY714, L896, and L897.

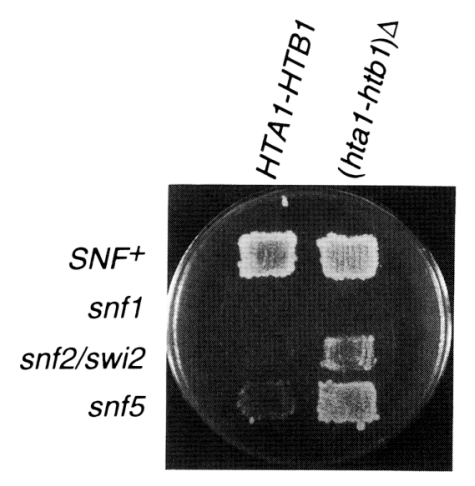

YPgal

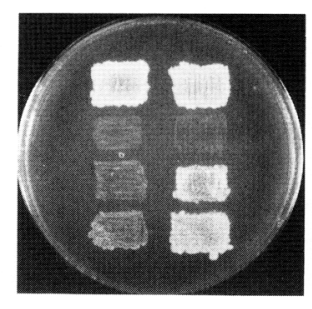

- inositol

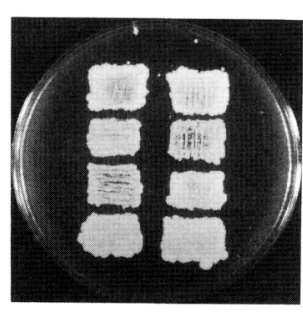

YPD



+ inositol
Figure 2. Suppression of growth defects of snf mutants by (hta1-htb1) $\Delta$. Strains were grown in patches on a YPD plate and then replica plated onto media containing galactose as a sole carbon source (YPgal), YPD, media lacking inositol (-inositol), and -inositol media supplemented with inositol (+inositol). Plates were photographed after 2 days of growth at $30^{\circ} \mathrm{C}$. The strains were as follows (from left to right): (Row 1) FY120 and FY710; (row 2) FY722 and FY723; (row 3) FY458 and FY724; (row 4) FY712 and FY714.

Consistent with these results, other studies have shown that snf6 mutations display somewhat different genetic interactions than do snf2 and snf5 mutations (Laurent et al. 1991; Laurent and Carlson 1992).

Many snf mutants also show growth defects on media containing galactose as a carbon source and on media lacking inositol (Neigeborn and Carlson 1984; Peterson et al. 1991). In the most extensively studied case, snf2 (swi2) mutants, these defects are probably the result of a reduced level of transcription of GAL10, GAL1, and INO1 (Peterson et al. 1991; Peterson and Herskowitz 1992). Therefore, we determined whether the $\mathrm{Gal}^{-}$and Ino- phenotypes of snf mutants are also suppressed by (hta1-htb1) $\Delta$. These results show that similar to the effects seen on SUC2 expression, (hta1-htb1) $\Delta$ suppresses these phenotypes in snf2, snf5, and snf6 mutants but not in snf1 mutants (Fig. 2; data not shown). Thus, a deletion of one of the two gene pairs encoding histones H2A and H2B suppresses multiple defects of snf2, snf5, and snf6 mutants.

\section{Mutations in SNF5 alter SUC2 chromatin structure}

The suppression of snf mutations by (hta1-htb1) $\Delta$ suggests that these SNF proteins activate transcription by altering chromatin structure. To test this possibility, we studied the chromatin structure of the SUC2 promoter 
region in $\mathrm{SNF5}^{+}$and snf5 mutant strains by examining sensitivity to cleavage by MNase. MNase cleavage of DNA is usually altered by nucleosomes (for review, see van Holde 1988); thus, differences in the pattern of MNase cleavage between different strains are likely to reflect differences in nucleosome position or structure.

To determine the sites of MNase sensitivity in the SUC2 promoter region, we isolated chromatin from four strains that were grown under derepressing conditions for SUC2 transcription (low glucose) and that differ only in their SNF5 and HTA1-HTB1 genotypes: wild type, snf5, (hta1-htb1) $\Delta$, and snf5 (hta1-htb1) $\Delta$. We then performed indirect end-labeling experiments (Nedospasov and Georgiev 1980; Wu 1980) as described in Materials and methods. In this technique, chromatin is subjected to partial digestion with MNase. DNA is then purified, digested to completion with a restriction enzyme, and subjected to Southern hybridization analysis, using a short probe immediately adjacent to one of the restriction enzyme sites. By this method, the positions of MNase cleavage sites in the SUC2 promoter region can be determined with respect to a defined restriction site.

The results of the indirect end-labeling experiments (Fig. 3) reveal that two regions of the SUC2 promoter show significant and reproducible differences in MNase cleavage patterns between different strains. First, at two sites near the TATA box, MNase cleaves both naked DNA and chromatin from a wild-type $\left(\mathrm{SNF}^{+}\right)$strain (Fig. 3 , sites $E$ and F). However, chromatin from a snf5 mutant is nearly completely protected from cleavage at these sites. This protection and the distance of $\sim 145 \mathrm{bp}$ between sites $D$ and $G$ are consistent with the presence of a nucleosome (diagramed in Fig. 6, below). The chromatin from a snf5 (hta1-htb1) $\Delta$ double mutant shows partial cleavage at sites $\mathrm{E}$ and $\mathrm{F}$ (Fig. 3, lanes 13-17). The effects of (hta1-htb1) $\Delta$ suggest that in a snf5 background, this histone mutation causes either a reduction or alteration of nucleosomes over the SUC2 promoter region, leading to increased transcription of SUC2.

Differences in chromatin structure also occur in a region between the SUC2 TATA box and upstream activation sequences (UAS). In this region, three adjacent MNase sites (Fig. 3, sites A, B, and C) are affected by snf5 and (hta1-htb1) $\Delta$ mutations. In naked DNA and in chromatin from wild-type strains, only sites $B$ and $C$ are cleaved. In contrast, chromatin from a snf5 strain exhibits protection from cleavage at site $\mathrm{C}$ and partial protection at site $\mathrm{B}$ and is sensitive to cleavage at site $\mathrm{A}$. The altered cleavage pattern in the snf5 mutant is also consistent with a nucleosome over this region: blockage of sites $\mathrm{B}$ and $\mathrm{C}$ and induced cutting adjacent to the putative nucleosome at site A (diagramed in Fig. 6). Chromatin from a snf5 (hta1-htb1) $\Delta$ double mutant shows partial cleavage at all three sites, again consistent with the suppression of snf5 by (hta1-htb1) $\Delta$.

\section{Alterations in chromatin structure do not require transcription or a functional TATA box}

Previous genetic results, combined with our results on
MNase cleavage of SUC2 chromatin, are consistent with the model that changes in chromatin structure at SUC2 cause transcriptional changes. However, these results are also consistent with the possibility of the reverse relationship: Changes in the level of transcription cause changes in chromatin structure. This latter possibility is supported by experiments showing that RNA polymerase-induced supercoiling during transcription can affect histone-DNA interactions (Liu and Wang 1987; Clark and Felsenfeld 1991; Lee and Garrard 1991) and that the TATA box-binding protein TFIID can compete with histones for DNA binding in vitro (Workman and Roeder 1987).

To address the cause-and-effect relationship between changes in transcription and chromatin structure at SUC2, we examined SUC2 chromatin structure under conditions where transcription was greatly reduced independently of SNF functions. To create these conditions, we mutated the SUC2 TATA box, generating the suc2-104 allele in which the TATA box at -133 (TATAAA) is replaced by a KpnI site (GGTACC). In an otherwise wild-type background, this mutation reduces SUC2 transcription by $\sim 20$-fold to a level at or below that measured for a snf5 mutant (Table 1).

We then examined MNase cleavage patterns in the suc2-104 mutant background and discovered that the TATA box mutation has virtually no effect on SUC2 chromatin structure, in spite of its strong effect on SUC2 transcription (Fig. 4; cf. with Fig. 3). In addition, chromatin from a snf5 (hta1-htb1) $\Delta$ strain carrying the suc2104 allele has a partly wild-type MNase cleavage pattern (Fig. 4, lanes 13-17), despite nearly undetectable levels of SUC2 expression (Table 1). Therefore, the level of SUC2 transcription does not determine SUC2 chromatin structure. Rather, the chromatin structure of the SUC2 promoter region is apparently determined by the presence or absence of SNF5 function.

Close comparison of Figures 3 and 4 reveals that MNase cleavage at a site near the TATA box (site F) is slightly diminished in chromatin from suc2-104 strains in comparison to chromatin from SUC2 strains. This difference is likely the result of the mutation of a favored MNase cleavage site (TATAAA; Dingwall et al. 1981; Hörz and Altenburger 1981) to a less preferred site (GGTACC), as naked DNA derived from a suc2-104 strain is also cleaved less efficiently at site $F$ than is naked DNA from a SUC2 strain (cf. Fig. 3, lane 2, with Fig. 4, lane 2). This difference in cleavage also suggests that site $F$ is located at the TATA box.

\section{Mutations in SNF2 also alter chromatin structure at SUC2}

We have shown that mutations in SNF5 cause changes in chromatin structure in the SUC2 promoter region. Because the SNF5 protein is thought to be functionally related to at least four other proteins, SNF2, SNF6, SWI1, and SWI3, we wanted to test whether a mutation in another member of this group would also cause changes in chromatin structure at SUC2. SNF2 is of particular in- 
Hirschorn et al.

Figure 3. snf5 mutants have altered chromatin structure over the SUC2 promoter region. Chromatin from the indicated strains was digested with differing amounts of MNase and subjected to indirect end-labeling analysis as described in Materials and methods. The probe used was in the $5^{\prime}$-coding region of $S U C 2$ adjacent to a HinfI site. Approximate positions of promoter elements (Sarokin and Carlson 1984) and MNase cleavage sites in the SUC2 promoter region are indicated at left; positions of size markers are indicated at right. Sources of DNA were as follows: Naked DNA from FY120 (lanes 1,2,23); chromatin from FY120 (lanes 3-7); chromatin from FY711 (lanes 8-12); chromatin from FY714 (lanes 13-17); and chromatin from FY710 (lanes 18-22). Chromatin from each strain was digested with $0,1,3,10$, or 30 units of MNase. Naked DNA was digested as follows: (Lanes 1,23) 0.3 units; (lane 2) 1 unit.

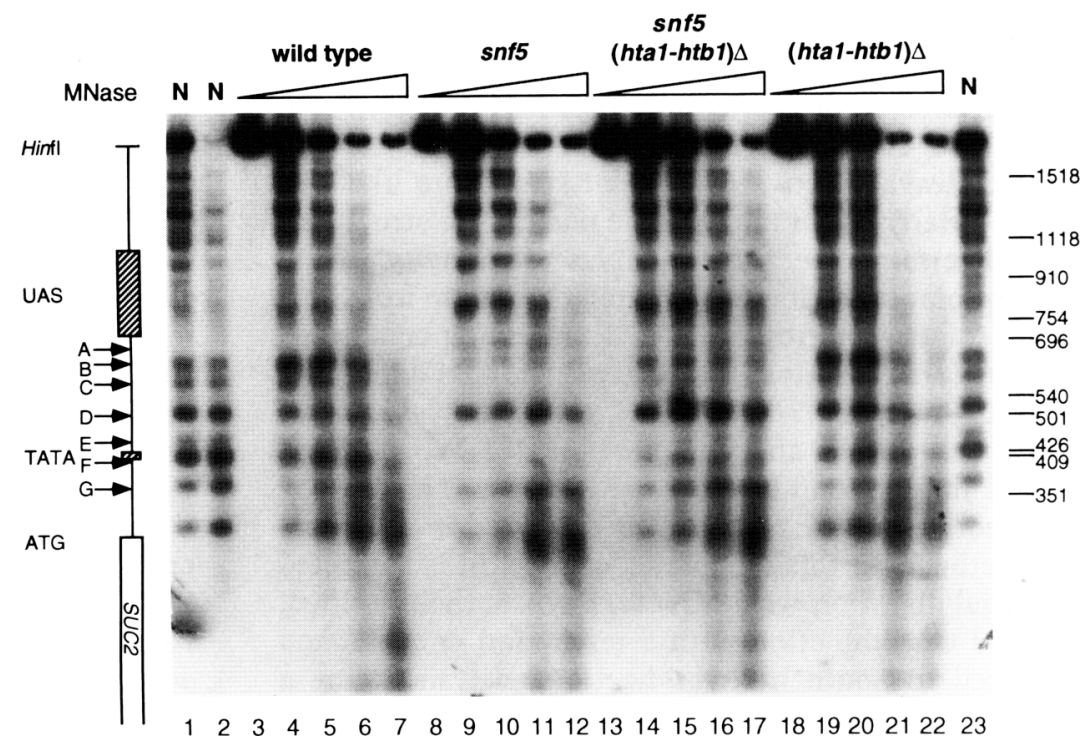

terest because it is evolutionarily conserved throughout eukaryotes and contains motifs found in helicases (Davis et al. 1992; Laurent et al. 1992; Tamkun et al. 1992). Therefore, we used indirect end-labeling to examine chromatin structure in snf2 and snf2 (hta1-htb1) $\Delta$ strains (Fig. 5).

Mutations in SNF2 cause essentially the identical changes in the chromatin structure of the SUC2 promoter region as do mutations in SNF5 /cf. Fig. 5 with Fig. 3). In particular, in snf2 mutants, sites B, C, E, and F are protected from cleavage, whereas cleavage at site $\mathrm{A}$ is induced. snf2 (hta1-htb1) $\Delta$ chromatin is nearly identical to snf5 (hta1-htb1) $\Delta$ chromatin; cleavage at sites B, C, E, and $F$ is partially restored, whereas cleavage at site $A$ is reduced. These data, combined with the other phenotypic similarities of snf2 and snf5 mutants, argue that
SNF2 and SNF5 play a similar role in activating transcription by altering chromatin structure.

\section{Discussion}

Our results indicate that SNF2 and SNF5 serve as transcriptional activators by overcoming the repressive effects of chromatin structure. This conclusion is based on three lines of evidence. First, mutations in these $S N F$ genes are suppressed by a deletion of one of the two loci encoding histones $\mathrm{H} 2 \mathrm{~A}$ and $\mathrm{H} 2 \mathrm{~B}$, (hta1-htb1) $\Delta$. Second, snf2 and snf5 mutants have altered chromatin structure at the SUC2 promoter; these alterations are partly reversed by (hta1-htb1) $\Delta$. Finally, a mutation of the SUC2 TATA box has no effect on the chromatin structure of the SUC2 promoter region, even though it reduces tran-
Figure 4. Mutation of the SUC2 TATA box does not affect chromatin structure at SUC2. Indirect end-labeling analysis was performed as described in the legend to Fig. 3. Sources of DNA were as follows: Naked DNA from FY716 (lanes 1,2,23); chromatin from FY716 (lanes 3-7); chromatin from FY719 (lanes 8-12); chromatin from FY721 (lanes 13-17); and chromatin from FY718 (lanes 18-22). All strains contained the TATA mutation suc2-104. Amounts of MNase were as described in the legend to Fig. 3, except lane 23 was digesed with 1 unit of MNase.

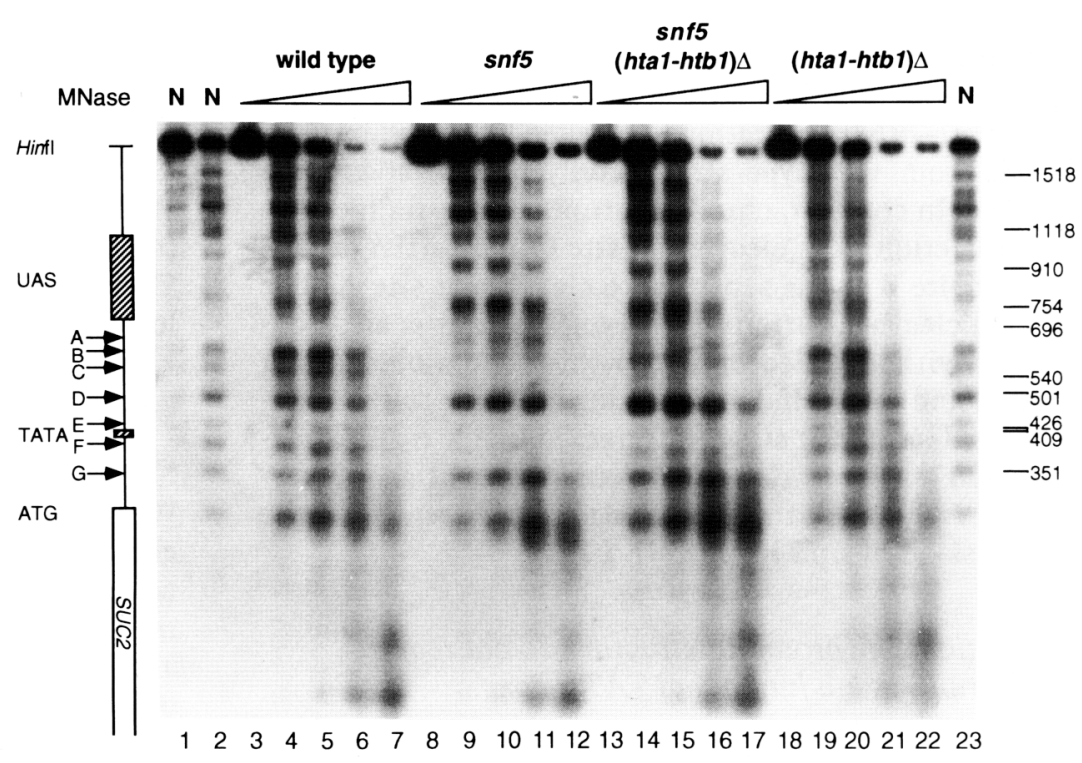




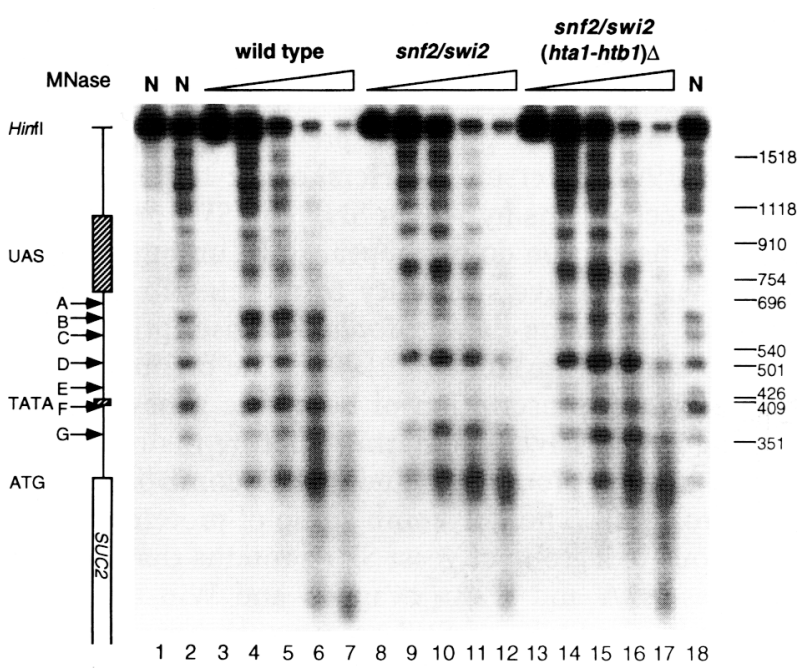

Figure 5. snf2 mutants have altered chromatin structure over the SUC2 promoter region. Indirect end-labeling analysis was performed as described in the legend to Fig. 3. Sources of DNA were as follows: Naked DNA from FY120 (lanes 1,2,18); chromatin from FY120 (lanes 3-7); chromatin from FY458 (lanes 9-12); chromatin from FY724 (lanes 13-17). Chromatin from each strain was digested with $0,1,3,10$, or 30 units of MNase. Naked DNA was digested as follows: (Lane 1) 0.3 units; (lanes 2,18) 1 unit.

scription of SUC2 to a level at or below that measured for a snf2 or snf5 mutant. Thus, the differences in the level of SUC2 transcription between wild-type and snf mutant strains are not responsible for the differences in chromatin structure between these strains. Rather, these results strongly suggest that SNF2 and SNF5 act directly on chromatin structure and that the resulting changes in chromatin structure cause changes in transcription.

MNase cleavage patterns provide the direct evidence that these SNF proteins activate transcription by altering chromatin structure. MNase has been shown to cleave preferentially the regions between nucleosomes; partial MNase digestion of bulk chromatin reveals a ladder of DNA fragments that are integral multiples of a nucleosome-sized fragment (for review, see van Holde 1988). Comparison of chromatin from different strains shows that in snf2 and snf5 mutants there is reproducibly much less cleavage by MNase at sites near the TATA box and between the TATA box and UAS than there is in $S N F^{+}$ strains. In addition, cleavage is induced in these mutants (possibly by the adjacent binding of a nucleosome) just $3^{\prime}$ to the UAS at a site that is not cut in naked DNA. Because these changes in snf mutants are mostly reversed by decreasing histone gene dosage, they are consistent with nucleosomes being present in snf2 and snf5 chromatin but absent, positioned differently, or otherwise modified in wild-type chromatin (Fig. 6). In addition, the distances between sites that are not protected from MNase cleavage in snf mutants are consistent with the size of a nucleosome.

Our data suggest that chromatin must be in an active configuration over the SUC2 promoter region for SUC2 transcription to occur. In this model, the SNF and SWI proteins establish or maintain an active chromatin structure so as to allow increased accessibility of the SUC2 promoter for binding of transcription factors. These could include general transcription factors (such as the TATA box-binding protein TFIID) and/or other as-yetunidentified proteins required for SUC2 transcription. One simple model that is consistent with our data is that the SNF and SWI activators function by removing or otherwise modifying nucleosomes to increase accessibility of the TATA box to TFIID (Fig. 6). This model is appealing because our results using strains without a functional SUC2 TATA box demonstrate that SNF-dependent alterations in chromatin structure do not require a functional TFIID-binding site, suggesting that these chromatin structure changes precede TFIID binding.

Recently, Matallana et al. (1992) also examined the chromatin structure at SUC2 in particular mutants, including snf2 and snf5 strains. Their general conclusions are consistent with ours (this paper and our unpublished data): Chromatin is altered in snf strains and appears similar in structure to the chromatin of wild-type cells grown under repressing conditions. However, we cannot make specific comparisons between the two sets of results, because many of the sites at which we observed alterations in MNase cleavage were not clearly detected in their experiments.

Previous studies suggest that the SNF and SWI proteins may have another role in activating transcription in addition to their effects on chromatin structure. These



(b) Active chromatin $\left(\mathrm{SNF}^{+} / \mathrm{SW} \mathrm{I}^{+}\right)$



Figure 6. A model for SNF2 and SNF5-dependent activation of SUC2 transcription. Possible chromatin structures of the SUC2 promoter region in different strains are depicted schematically. Approximate locations of MNase cleavage sites in chromatin from a snf2 or snf5 mutant $(a)$ and from a $S N F^{+}$strain $(b)$ are indicated by the arrows below each line; a small arrow represents reduced cleavage by MNase. The solid ovals represent nucleosomes; the broken ovals represent nucleosomes that have been removed or modified so as to increase accessibility of the SUC2 promoter region. SNF and SWI proteins could help in either establishment or maintenance of the chromatin structure shown in $b$. 
studies showed that LexA-SNF hybrid proteins can activate transcription of a reporter gene whose promoter contains a lexA operator (Laurent et al. 1990, 1991; Laurent and Carlson 1992), indicating that SNF proteins may also activate transcription by directly stimulating the transcription machinery. However, these results are also consistent with the possibility that SNF proteins activate transcription solely by altering chromatin structure, because the lex $A$ reporter plasmid is almost certainly assembled into chromatin in the cell. By this model, LexA-SNF hybrid proteins could activate transcription by changing the chromatin structure of the reporter plasmid to an active configuration that is accessible to the transcriptional machinery. More detailed biochemical studies of SNF and SWI proteins will be required to distinguish between these and other possibilities.

SNF2 and SNF5 both contain regions that could directly modulate chromatin structure. SNF2 encodes an evolutionarily conserved putative helicase; helicase activity could affect nucleosome stability, perhaps through local changes in supercoiling. This possibility is consistent with models discussed by Travers (1992). SNF5 encodes a protein that is glutamine- and proline-rich (Laurent et al. 1990), a characteristic of some transcriptional activators (Mitchell and Tjian 1989). Spl, which has been shown to overcome repression by chromatin in vitro (Croston et al. 1991), requires a glutamine-rich domain to activate transcription (Courey and Tjian 1988). Therefore, glutamine- and proline-rich regions may function as activation domains by altering chromatin structure.

The precise sites and mechanisms of action of the SNF and SWI proteins at the SUC2 promoter are unknown. SNF and SWI proteins may activate transcription by directly altering chromatin structure between the UAS and the TATA box and over the TATA box. However, the changes observed in either of these regions could be indirect effects of other proteins binding to the SUC2 promoter region. For example, the SNF and SWI proteins might enable the binding of an as-yet-unidentified activator to the chromatin in the SUC2 UAS, either by altering chromatin structure in the UAS or by increasing the binding affinity of this activator for nucleosomes. The binding of this activator might then alter chromatin structure in adjacent promoter regions, in a manner similar to the effect of GRF2 binding to the GAL1-GAL10 intergenic region (Fedor et al. 1988).

Other proteins have been proposed to activate transcription by increasing the accessibility of promoter regions for transcription factors. For example, Fascher et al. (1990) have shown that activation of PHO5 transcription may involve removal of nucleosomes by the PHO4 transcriptional activator. Because SNF and SWI proteins are also required for $\mathrm{PHO} 5$ expression (Abrams et al. 1986), PHO4 may function by recruiting SNF and SWI proteins to the $\mathrm{PHO} 5$ promoter region, where they could cause changes in chromatin structure. Alternatively, SNF and SWI proteins may interact with the $\mathrm{PHO} 4$ protein or alter the $\mathrm{PHO} 5$ chromatin structure to allow the binding of $\mathrm{PHO} 4$ to nucleosomes in the $\mathrm{PHO} 5$ promoter region. Similar roles for SNF and SWI proteins in aiding many different gene-specific transcriptional activators to function have been proposed previously (Laurent and Carlson 1992; Peterson and Herskowitz 1992).

The mechanisms by which SNF and SWI proteins activate transcription do not appear to be unique to yeast. SNF2 has extensive similarity to proteins in other eukaryotes, including the Drosophila transcriptional activator brahma (Tamkun et al. 1992). The brahma protein may activate transcription of homeotic genes by overcoming a repressive chromatin structure maintained by a large group of proteins, including Polycomb (for review, see Paro 1990). The Polycomb group of proteins may be analogous to a group of yeast SPT proteins that includes histones H2A and H2B (Swanson and Winston 1992). Thus, like the SNF and SWI proteins, brahma may activate transcription by overcoming repression mediated by chromatin. Finally, the Drosophila transcriptional activators fushi tarazu and Bicoid both require SNF and SWI proteins to function in yeast (Laurent and Carlson 1992; Peterson et al. 1992), further suggesting the presence of SNF and SWI functional homologs in other organisms.

One appealing possible role for SNF and SWI proteins would be to remove one or both $\mathrm{H} 2 \mathrm{~A}-\mathrm{H} 2 \mathrm{~B}$ dimers from nucleosomes, perhaps leaving an $\mathrm{H} 3-\mathrm{H} 4$ tetramer bound to the DNA. This possibility is particularly intriguing because snf/swi mutations can be suppressed by a deletion of one of the two gene pairs encoding histones H2A and $\mathrm{H} 2 \mathrm{~B}$, which probably results in lower levels of these histones in vivo. Dong and van Holde (1991) have shown that $\mathrm{H} 3-\mathrm{H} 4$ tetramers can be positioned on DNA in a manner similar to intact nucleosomes and that MNase cleavage of tetramer-bound DNA is enhanced relative to cleavage of nucleosomes. In addition, Baer and Rhodes (1983) have shown that RNA polymerase binds preferentially to nucleosomes that are deficient in $\mathrm{H} 2 \mathrm{~A}$ and $\mathrm{H} 2 \mathrm{~B}$. Finally, histone octamers, but not $\mathrm{H} 3-\mathrm{H} 4$ tetramers, efficiently repress 5S RNA transcription in vitro (Tremethick et al. 1990; Almouzni et al. 1991). This model also raises the possibility that proteins functionally related to histones, such as the acidic SPT5 and SPT6 proteins (Swanson et al. 1990, 1991), could repress transcription by stabilizing the interaction of $\mathrm{H} 2 \mathrm{~A}-\mathrm{H} 2 \mathrm{~B}$ dimers with DNA-bound $\mathrm{H} 3-\mathrm{H} 4$ tetramers, in a manner analogous to that of the acidic chromatin assembly factor nucleoplasmin (Dingwall et al. 1987). Other proteins implicated in affecting chromatin structure, including SIN1/SPT2, SPT4, and SPT16 (Kruger and Herskowitz 1991; Malone et al. 1991; Swanson and Winston 1992), might also affect histone-histone or histone-DNA interactions.

In conclusion, we have presented genetic and molecular data strongly suggesting that the yeast SNF2 and SNF5 transcriptional activators function by causing changes in chromatin structure. Because these SNF and SWI proteins control transcription of many different genes in yeast, this mechanism of activation appears to be widespread. Further genetic, molecular, and biochemical analyses will be necessary to elucidate the precise functions of these proteins and of other proteins that 
may regulate chromatin structure. Finally, the strong evolutionary conservation of SNF2 and of histones indicates that an understanding of the mechanism by which these proteins control gene expression in yeast will contribute to the understanding of gene expression throughout eukaryotes.

\section{Materials and methods \\ Strains and genetic methods}

Yeast strains are listed in Table 2 and are isogenic to the strain S288C, except that they are $G A L 2^{+}$(C. Dollard and F. Winston, unpubl.). Uppercase letters denote the dominant (wild-type) allele, and lowercase letters denote the recessive (mutant) allele. Standard methods for mating, sporulation, and tetrad analysis were used (Rose et al. 1990). A strain containing the (hta1htb1| $\Delta:: L E U 2$ allele was constructed by transformation with the 3.8-kb BamHI-NheI fragment of $\mathrm{pJH} 8$. Leu ${ }^{+}$transformants were screened for suppression of the insertion mutations his4$912 \delta$ and lys2-128 $\delta$. A strain containing the suc2-101::URA3 allele was constructed by transformation with the $2.2-\mathrm{kb}$ Hin dIII fragment of pJH38. A strain containing the suc2-104 allele was constructed by transforming strain FY637 with the $0.9-\mathrm{kb}$ EcoRI-HindIII fragment of $\mathrm{pJH} 42$ and selecting for resistance to 5 -fluoro-orotic acid. Strains carrying the snf $4 \Delta 1$ (Celenza et al. 1989) and snf642 (Estruch and Carlson 1990) null alleles were kindly provided by M. Carlson (Columbia University, New York). Strains carrying the snf1 $1 \Delta 10$, snf2 $21:: H I S 3$, and snf55::URA3 null alleles were constructed as described (Abrams et al. 1986; Celenza and Carlson 1989). During the course of these studies, we noticed that snf5 mutants occasionally acquired weak second-site suppressors (J. Hirschhorn and F. Winston,

Table 2. S. cerevisiae strains

\begin{tabular}{|c|c|}
\hline Strain & Relevant genotype $^{a}$ \\
\hline FY56 & $M A T \alpha$ \\
\hline FY120 & MATa \\
\hline FY710 & MATa $(h t a 1-h t b 1) \Delta:: L E U 2$ \\
\hline FY738 & MATa $($ hta1-htb1) $\Delta:: L E U 2$ \\
\hline FY722 & MATa snf $1 \Delta 10$ \\
\hline FY723 & MATa snf1 $110($ hta1-htb1) $\Delta:: L E U 2$ \\
\hline FY737 & MATa snf2 $21: \because H I S 3$ his $3 \Delta 200$ his $3 \Delta 200$ \\
\hline FY458 & MATa snf $2 \Delta 1:: H I S 3$ \\
\hline FY724 & MATa snf2A1::HIS3 (hta1-htb1) $\Delta:: L E U 2$ \\
\hline L898 & MATa $\operatorname{snf} 4 \Delta 1$ \\
\hline L899 & MATa snf $4 \Delta 1$ (hta1-htb1) $\Delta:: L E U 2$ \\
\hline FY711 & $M A T \alpha$ snf5-5::URA3 \\
\hline FY712 & $M A T \mathbf{a}$ snf5-5::URA3 \\
\hline FY713 & MAT $\alpha$ snf5-5::URA3 (hta1-htb1) $\Delta:: L E U 2$ \\
\hline FY714 & MATa snf5-5::URA3 (hta1-htb1) $\Delta:: L E U 2$ \\
\hline L896 & $M A T \mathbf{a}$ snf $6 \Delta 2$ \\
\hline L897 & MATa snf6 $62(h t a 1-h t b 1) \Delta:: L E U 2$ \\
\hline FY637 & MATa suc2-101::URA3 \\
\hline FY716 & MAT $\alpha$ suc2-104 \\
\hline FY718 & MATa suc2-104 (hta1-htb1) $\Delta:: L E U 2$ \\
\hline FY719 & MATa suc2-104 snf5-5::URA3 \\
\hline FY721 & MATa suc2-104 snf5-5::URA3 (hta1-htb1) $\Delta::$ LEU2 \\
\hline
\end{tabular}

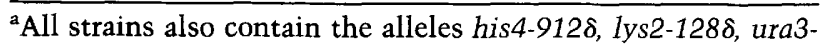
52, and leu2A1, except for FY56 and FY458, which are LEU2, and FY737, which is HIS4. All strains were constructed for this study. unpubl.). These suppressors were detected by weak suppression of the insertion mutations his4-912 $\delta$ and $1 y s 2-128 \delta$ and had no detectable effect on MNase cleavage patterns (J. Hirschhorn and F. Winston, unpubl.). Nevertheless, the snf5 strains used in this study were put through crosses to confirm the absence of additional mutations and were carefully monitored to ensure that phenotypes of strains grown in cultures were unchanged. For all alleles constructed in this study, transformants were shown to be correct by Southern hybridization analysis.

Yeast strains were transformed by the lithium acetate method as described (Rose et al. 1990). Escherichia coli HB101 (Ausubel et al. 1988), TB1 (Bethesda Research Laboratories, Gaithersburg, MD), and GM237 (Arraj and Marinus 1983) were transformed as described (Ausubel et al. 1988).

Media

All media were made as described (Rose et al. 1990). YEP plus $0.05 \%$ glucose was identical to YPD except that the media contained $0.05 \%$ glucose instead of $2 \%$ glucose as the carbon source. YPsuc, YPraff, and YPgal media contained 2\% sucrose, raffinose, or galactose, respectively, as the sole carbon source and contained $1 \mathrm{mg} /$ liter of antimycin A. Solid media lacking inositol were made as described (Sherman et al. 1978).

\section{Enzymes and protease inhibitors}

Restriction enzymes, T4 DNA ligase, DNA polymerase I (Klenow fragment) and Taq polymerase were purchased from Boehringer Mannheim Biochemicals (Indianapolis, IN) and from New England BioLabs (Beverly, MA). MNase was purchased from Boehringer Mannheim Biochemicals and stored at 6000 $\mathrm{U} / \mathrm{ml}$ at $-20^{\circ} \mathrm{C}$ in $50 \%$ glycerol, $2 \mathrm{mM} \mathrm{CaCl}_{2}$, and $50 \mathrm{mM}$ Tris $(\mathrm{pH} 9.0)$. All enzymes were used according to the instructions of the supplier. All protease inhibitors were purchased from Sigma (St. Louis, MO), and stocks were prepared and stored according to the instructions of the supplier.

\section{DNA preparation and analysis}

Yeast genomic DNA (Hoffman and Winston 1987) and plasmids from E. coli (Ausubel et al. 1988) were prepared as described previously. Analysis and purification of DNA restriction fragments were performed as described previously (Swanson et al. 1991). Southern blot hybridization analysis was performed as described in the instruction manual for GeneScreen (New England Nuclear, Boston, MA) using the dextran sulfate method.

\section{Plasmids}

The construction of a plasmid for generation of the (hta1$h t b 1 \mid \Delta:: L E U 2$ allele involved several steps. First, the HindIII site in pUCl 8 was changed to an NheI site by digestion with HindIII, filling in with Klenow, and religation, creating the plasmid pJH1. pJH1 was digested with BamHI and HincII, and a 1.6-kb BamHI-HpaI HTA1-HTB1 fragment from pCC72 (C. Clark and F. Winston, unpubl.) was inserted, creating plasmid pJH3. This HTA1-HTB1 fragment extends from the HpaI site $3^{\prime}$ to HTA1 to the BamHI site $3^{\prime}$ to HTB1 but lacks the $1.1-\mathrm{kb}$ HindIII promoter-containing fragment and $329 \mathrm{bp}$ immediately adjacent to the BamHI site (M. Osley, pers. comm.). After passage through the $E$. coli strain GM237 $\left(\right.$ dam $\left.^{-}\right)$, pJH3 was digested with $X b a \mathrm{I}$ and HindIII, and the $L E U 2$ gene was inserted into these sites, creating plasmid pJH8. For this insertion of $L E U 2$, a 2.2-kb SalI-XhoI fragment from YEp13 containing the $L E U 2$ gene was first cloned into the Sall site of a derivative of 
pUC18 (provided by D. Eisenmann, Stanford University, CA) and then excised by digestion with $\mathrm{XbaI}$ and HindIII. For construction of the suc2-101::URA3 allele, the 1.1-kb HindIII fragment containing the SUC2 promoter from pRB58 (Carlson and Botstein 1982) was cloned into pUC18, creating plasmid pJH35. pJH35 was then digested with MluI and NcoI, and a 1.1-kb Hin. dIII fragment containing URA3 was inserted between these sites after all sites were filled in with Klenow, creating plasmid $\mathrm{pJH} 38$. For construction of the suc2-104 allele, the $0.9-\mathrm{kb}$ EcoRI-HindIII SUC2 promoter fragment of pRB58 was cloned into mp19, and the TATA box at - 133 (TATAAA) was changed to a $K p n I$ site (GGTACC) by site-directed mutagenesis (Kunkel 1985) using the oligonucleotide 5 '-CCTTCCTCCTGAAAAGAAACAGGTACCTAGATATGTATTATTCTTC. The resulting EcoRI-HindIII fragment was cloned into pUC19, creating plasmid $\mathrm{pJH} 42$.

\section{Hybridization probes}

Plasmids used as probes for RNA analysis were as follows: for Ty, B161 (Happel et al. 1991); for TUB2, pYST138 (Som et al. 1988); for TPI1, pHB59 (H. Baker, pers. comm.). Probes used for RNA analysis and indirect end-labeling of SUC2 were DNA fragments generated using the polymerase chain reaction (PCR) essentially as described (Ausubel et al. 1988) with pRB58 as a template. For mRNA analysis, the probe extended from +787 to +966 , and for indirect end-labeling, the probe extended from +140 to +296 , relative to the start of translation. ${ }^{32} \mathrm{P}$-Labeled plasmids were prepared by nick translation using a kit from Boehringer Mannheim Biochemicals. ${ }^{32} \mathrm{P}$-Labeled DNA fragments were prepared by primer extension with Klenow essentially as described for radiolabeling DNA fragments using random hexamers (Ausubel et al. 1988), except that 50 pmoles each of the two oligonucleotides that had been used for PCR were used as primers.

\section{RNA preparation and analysis}

For analysis of Ty mRNA, yeast RNA was prepared as described previously (Carlson and Botstein 1982) from cells grown in YPD to a density of $1 \times 10^{7}$ to $2 \times 10^{7}$ cells $/ \mathrm{ml}$. For analysis of SUC2 mRNA, cells were grown in $10 \mathrm{ml}$ of YPD to a density of $6 \times 10^{6}$ to $1 \times 10^{7}$ cells $/ \mathrm{ml}$, washed twice with $10 \mathrm{ml}$ of water, and grown in $10 \mathrm{ml}$ of YEP plus $0.05 \%$ glucose for $2.75 \mathrm{hr}$. Electrophoresis, transfer to GeneScreen (New England Nuclear), and hybridization analysis were performed as described previously (Swanson et al. 1991), except that for SUC2 mRNA analysis, the wash conditions were two washes of $250 \mathrm{ml} 2 \times$ SSC at $23^{\circ} \mathrm{C}$ for $15 \mathrm{~min}$, followed by two washes of $250 \mathrm{ml} 2 \times \mathrm{SSC}$, $0.5 \% \mathrm{SDS}$, at $57^{\circ} \mathrm{C}$ for $15 \mathrm{~min}$. The amount of RNA in each lane was standardized by hybridization to PYST138 containing TUB2 or to pHB59 containing TPI1.

\section{Invertase assays}

Secreted invertase activity was determined by assaying whole cells (Goldstein and Lampen 1975), as described previously by Celenza and Carlson (1984). Glucose-repressed cultures were grown in YPD at $30^{\circ} \mathrm{C}$ to a density of $6 \times 10^{6}$ to $1 \times 10^{7}$ cells/ $\mathrm{ml}$. Derepressed cultures were prepared by washing these cultures twice with water, shifting to an equal volume of YEP plus $0.05 \%$ glucose, and growing at $30^{\circ} \mathrm{C}$ for $2.75 \mathrm{hr}$. For calculation of units of activity, cell number was converted to dry weight of cells; $1 \times 10^{7}$ cells are equivalent to $0.38 \mathrm{mg}$ of cells, dry weight (Swanson and Winston 1992).

\section{Preparation of yeast chromatin}

Chromatin was prepared using a modified version of a protocol provided by B. Dunn (pers. comm.) One liter of cells was grown in YPD at $30^{\circ} \mathrm{C}$ to a concentration of $6 \times 10^{6}$ to $1 \times 10^{7}$ cells/ $\mathrm{ml}$. Cultures were washed twice with $330 \mathrm{ml}$ of water and resuspended in 1 liter of YEP plus $0.05 \%$ glucose. After $2.75 \mathrm{hr}$ of further growth at $30^{\circ} \mathrm{C}$ to relieve glucose repression, cells were washed with $330 \mathrm{ml}$ of water and the cell pellet was weighed. Cells were washed with $30 \mathrm{ml}$ of cold DTT solution $110 \mathrm{~mm}$ DTT, $20 \mathrm{~mm}$ potassium phosphate at $\mathrm{pH} 7.0,1 \mathrm{M}$ Sorbitol) and resuspended in $4 \mathrm{ml} / \mathrm{gm}$ cell weight of $S$ buffer $(1.1 \mathrm{M}$ Sorbitol, $20 \mathrm{mM}$ potassium phosphate at $\mathrm{pH} 7.0,0.5 \mathrm{mM} \mathrm{CaCl}_{2}$ / containing $1 \mathrm{mg} / \mathrm{ml}$ of Zymolyase T100 $(50,000 \mathrm{U} / \mathrm{mg})$ and $0.5 \mathrm{~mm}$ phenylmethylsulfonyl fluoride (PMSF; Sigma). Cells were shaken at $95 \mathrm{rpm}$ at $30^{\circ} \mathrm{C}$ for $35 \mathrm{~min}$; $>95 \%$ of cells were converted to spheroplasts as monitored by lysis in a 50 -fold excess of water. All subsequent steps were performed at $4^{\circ} \mathrm{C}$, and all buffers contained protease inhibitors as described by Norris et al. (1988). SPC and Ficoll buffers are as described by Nelson and Fangman (1979). Spheroplasts were harvested by centrifugation in a Sorvall SA600 rotor (DuPont, Wilmington, DE) at $5000 \mathrm{rpm}$ for $5 \mathrm{~min}$ and washed with $15 \mathrm{ml}$ of SPC buffer. Spheroplasts were gently resuspended in $0.5 \mathrm{ml}$ of SPC buffer and lysed by addition to $25 \mathrm{ml}$ of Ficoll buffer with gentle stirring over $15 \mathrm{sec}$. The solution was poured back into the original tube and allowed to settle for $10 \mathrm{~min}$. "Supernatant" $(23.5 \mathrm{ml})$ was transferred to a new tube and centrifuged in an SA600 rotor at 13,200 rpm for $20 \mathrm{~min}$. The pellet was resuspended in $17 \mathrm{ml}$ of SPC buffer and recentrifuged in an SA600 rotor at $10,100 \mathrm{rpm}$ for $10 \mathrm{~min}$. The pellet, which contains nuclei, was resuspended in $2 \mathrm{ml} / \mathrm{gm}$ cell weight of SPC buffer, frozen on dry ice, and stored at $-70^{\circ} \mathrm{C}$.

\section{Indirect end-labeling analysis of yeast chromatin}

MNase (0, 1, 3, 10, or 30 units) was added to $200 \mu$ l of nuclei and incubated at $37^{\circ} \mathrm{C}$ for $10 \mathrm{~min}$. For naked DNA controls, yeast genomic DNA from $\sim 8 \mathrm{ml}$ of a saturated culture was brought to $1 \mathrm{M}$ Sorbitol, $20 \mathrm{mM}$ PIPES (pH 6.3), and $10 \mathrm{mM} \mathrm{CaCl}_{2}$ (SPC plus $10 \mathrm{mM} \mathrm{CaCl}_{2}$ ) in a final volume of $200 \mu \mathrm{l}$ before incubation with 0.3 or 1 unit of $\mathrm{MNase}$ at $37^{\circ} \mathrm{C}$ for $10 \mathrm{~min}$. The excess $\mathrm{CaCl}_{2}$ was required to overcome the chelation of divalent cations by DNA that had been treated with EDTA. Reactions were stopped by the addition of $40 \mu \mathrm{l}$ of $5 \mathrm{M} \mathrm{NaCl}, 8 \mu \mathrm{l}$ of $0.5 \mathrm{M}$ EDTA (pH 8.0), and $20 \mu \mathrm{l}$ of $10 \%$ SDS. DNA was purified as described by Bloom and Carbon (1982), except that an additional extraction with chloroform/isoamyl alcohol was performed before the first ethanol precipitation and DNA was resuspended in $20 \mu \mathrm{l}$ of TE at the end. For subsequent restriction digestion and Southern analysis, $9 \mu \mathrm{l}$ was used; electrophoresis was through $2 \%$ Seakem ME agarose (FMC BioProducts, Rockland, ME), and subsequent steps were performed as according to the dextran sulfate method of the GeneScreen manual, except that filters were washed three to four times for $15 \mathrm{~min}$ with $500 \mathrm{ml}$ of $2 \times$ SSC, $1 \%$ SDS, at $57^{\circ} \mathrm{C}$. For the experiments shown, the restriction enzyme HinfI was used; essentially identical results were obtained when different restriction enzymes and probes were used to study MNase cleavage patterns (data not shown).

\section{Acknowledgments}

We thank David Altshuler, Alex Bortvin, Lisa Gansheroff, Greg Prelich, and Lena Wu for useful discussions and critical readings of the manuscript. We thank Catherine Dollard for integration of the $\operatorname{snf1,} s n f 2$, and snf5 alleles. We are grateful to Marian Carlson for strains and plasmids and to Mary Ann Osley and 
Henry Baker for plasmids. This work was supported by National Institutes of Health grant GM32967 and a grant from the Stroh Brewing Company to F.W. J.H. was partially supported by the Medical Scientist Training Program (National Institutes of Health grant 2 T32 GM07753 to the Harvard Medical School M.D.-Ph.D. program).

The publication costs of this article were defrayed in part by payment of page charges. This article must therefore be hereby marked "advertisement" in accordance with 18 USC section 1734 solely to indicate this fact.

\section{References}

Abrams, E., L. Neigeborn, and M. Carlson. 1986. Molecular analysis of SNF2 and SNF5, genes required for expression of glucose-repressible genes in Saccharomyces cerevisiae. Mol. Cell. Biol. 6: 3643-3651.

Almouzni, G., M. Méchali, and A.P. Wolffe. 1991. Transcription complex disruption caused by a transition in chromatin structure. Mol. Cell. Biol. 11: 655-665.

Arraj, J.A. and M.G. Marinus. 1983. Phenotypic reversal in dam mutants of the Escherichia coli K-12 by a recombinant plasmid containing the dam ${ }^{+}$gene. I. Bacteriol. 153: 562-565.

Ausubel, F.M., R. Brent, R.E. Kingston, D.D. Moore, J.G. Seidman, J.A. Smith, and K. Struhl. 1988. Current protocols in molecular biology. Greene Publishing Associates/Wiley-Interscience, New York.

Baer, B.W. and D. Rhodes. 1983. Eukaryotic RNA polymerase II binds to nucleosome cores from transcribed genes. Nature 301: 482-488.

Bloom, K.S. and J. Carbon. 1982. Yeast centromere DNA is in a unique and highly ordered structure in chromosomes and small circular minichromosomes. Cell 29: 305-317.

Carlson, M. 1987. Regulation of sugar utilization in Saccharomyces species. J. Bacteriol. 169: 4873-4877.

Carlson, M. and D. Botstein. 1982. Two differentially regulated mRNAs with different 5 ' ends encode secreted and intracellular forms of yeast invertase. Cell 28: 145-154.

Carlson, M., B.C. Osmond, and D. Botstein. 1981. Mutants of yeast defective in sucrose utilization. Genetics 98: 25-40.

Celenza, J.L. and M. Carlson. 1984. Cloning and genetic mapping of $S N F 1$, a gene required for expression of glucose-repressible genes in Saccharomyces cerevisiae. Mol. Cell. Biol. 4: 49-53.

-1989. Mutational analysis of the Saccharomyces cerevisiae SNF1 protein kinase and evidence for functional interaction with the SNF4 protein. Mol. Cell. Biol. 9: 5034 5044.

Celenza, J.L., F.J. Eng, and M. Carlson. 1989. Molecular analysis of the SNF4 gene of Saccharomyces cerevisiae: Evidence for physical association of the SNF4 protein with the SNF1 protein kinase. Mol. Cell. Biol. 9: 5045-5054.

Ciriacy, M., K. Freidel, and C. Löhning. 1991. Characterization of trans-acting mutations affecting $\mathrm{Ty}$ and Ty-mediated transcription in Saccharomyces cerevisiae. Curr. Genet. 20: $441-448$.

Clark, D.J. and G. Felsenfeld. 1991. Formation of nucleosomes on positively supercoiled DNA. EMBO J. 10: 387-395.

Clark-Adams, C.D. 1988. "Effects on gene expression caused by mutation and altered dosage of the yeast SPT6 and histone genes." Ph.D. thesis, Harvard University, Cambridge, MA.

Clark-Adams, C.D., D. Norris, M.A. Osley, J.S. Fassler, and F. Winston. 1988. Changes in histone gene dosage alter transcription in yeast. Genes \& Dev. 2: 150-159.

Courey, A.J. and R. Tjian. 1988. Analysis of $\mathrm{Spl}$ in vivo reveals multiple transcriptional domains, including a novel glutamine-rich activation motif. Cell 55: 887-898.

Croston, G.E., L.A. Kerrigan, L.M. Lira, D.R. Marshak, and J.T. Kadonaga. 1991. Sequence-specific antirepression of histone H1-mediated inhibition of basal RNA polymerase II transcription. Science 251: 643-649.

Davis, J.L., R. Kunisawa, and J. Thorner. 1992. A presumptive helicase (MOT1 gene product) affects gene expression and is required for viability in the yeast Saccharomyces cerevisiae. Mol. Cell. Biol. 12: 1879-1892.

Dingwall, C., G.P. Lomonossoff, and R.A. Laskey. 1981. High sequence specificity of micrococcal nuclease. Nucleic Acids Res. 9: 2659-2673.

Dingwall, C., S.M. Dilworth, S.J. Black, S.E. Kearsey, L.S. Cox, and R.A. Laskey. 1987. Nucleoplasmin cDNA sequence reveals polyglutamic acid tracts and a cluster of sequences homologous to putative nuclear localization signals. EMBO J. 6: 69-74.

Dong, F. and K.E. van Holde. 1991. Nucleosome positioning is determined by the $(\mathrm{H} 3-\mathrm{H} 4)_{2}$ tetramer. Proc. Natl. Acad. Sci. 88: 10596-10600.

Estruch, F. and M. Carlson. 1990. SNF6 encodes a nuclear protein that is required for expression of many genes in Saccharomyces cerevisiae. Mol. Cell. Biol. 10: 2544-2553.

Fascher, K.-D., J. Schmitz, and W. Hörz. 1990. Role of transactivating proteins in the generation of active chromatin at the $P H O 5$ promoter in $S$. cerevisiae. EMBO I. 9: 2523-2528.

Fassler, J.S. and F. Winston. 1988. Isolation and analysis of a novel class of suppressor of Ty insertion mutations in Saccharomyces cerevisiae. Genetics 118: 203-212.

Fedor, M.J., N.F. Lue, and R.D. Kornberg. 1988. Statistical positioning of nucleosomes by specific protein-binding to an upstream activating sequence in yeast. I. Mol. Biol. 204: 109-127.

Goldstein, A. and J.O. Lampen. 1975. $\beta$-D-Fructofuranoside fructohydrolase from yeast. Methods Enzymol. 42: 504-511.

Grunstein, M. 1990a. Histone function in transcription. Annu. Rev. Cell Biol. 6: 643-678.

- 1990b. Nucleosomes: Regulators of transcription. Trends Genet. 6: 395-400.

Happel, A.M., M.S. Swanson, and F. Winston. 1991. The SNF2, SNF5 and SNF6 genes are required for Ty transcription in Saccharomyces cerevisiae. Genetics 128: 69-77.

Hoffman, C.S. and F. Winston. 1987. A ten-minute DNA preparation from yeast efficiently releases autonomous plasmids for transformation of Escherichia coli. Gene 57: 267-272.

Hörz, W. and W. Altenburger. 1981. Sequence specific cleavage of DNA by micrococcal nuclease. Nucleic Acids Res. 9: $2643-2658$.

Kruger, W. and I. Herskowitz. 1991. A negative regulator of $H O$ transcription, SIN1 (SPT2), is a nonspecific DNA-binding protein related to HMG1. Mol. Cell. Biol. 11: 4135-4146.

Kunkel, T.A. 1985. Rapid and efficient site-specific mutagenesis without phenotypic selection. Proc. Natl. Acad. Sci. 82: 488-492.

Laurent, B.C. and M. Carlson. 1992. Yeast SNF2/SWI2, SNF5, and SNF6 proteins function coordinately with the gene-specific transcriptional activators GAL4 and Bicoid. Genes \& Dev. 6: 1707-1715.

Laurent, B.C., M.A. Treitel, and M. Carlson. 1990. The SNF5 protein of Saccharomyces cerevisiae is a glutamine- and proline-rich transcriptional activator that affects expression of a broad spectrum of genes. Mol. Cell. Biol. 10: 5616-5625.

Laurent, B.C., M.A. Treitel, and M. Carlson. 1991. Functional interdependence of the yeast SNF2, SNF5, and SNF6 proteins in transcriptional activation. Proc. Natl. Acad. Sci. 
Hirschorn et al.

88: 2687-2691.

Laurent, B.C., X. Yang, and M. Carlson. 1992. An essential Saccharomyces cerevisiae gene homologous to SNF2 encodes a helicase-related protein in a new family. Mol. Cell. Biol. 12: 1893-1902.

Lee, M.-S. and W.T. Garrard. 1991. Transcription-induced nucleosome "splitting": An underlying structure for DNase I sensitive chromatin. EMBO J. 10: 607-615.

Liu, L.F. and J.C. Wang. 1987. Supercoiling of the DNA template during transcription. Proc. Natl. Acad. Sci. 84: 7024-7027.

Malone, E.A., C.D. Clark, A. Chiang, and F. Winston. 1991. Mutations in SPT16/CDC68 suppress cis- and trans-acting mutations that affect promoter function in Saccharomyces cerevisiae. Mol. Cell. Biol. 11: 5710-5717.

Matallana, E., L. Franco, and J.E. Pérez-Ortín. 1992. Chromatin structure of the yeast SUC2 promoter in regulatory mutants. Mol. Gen. Genet. 231: 395-400.

Mitchell, P.J. and R. Tiian. 1989. Transcriptional regulation in mammalian cells by sequence-specific DNA binding proteins. Science 245: 371-378.

Nedospasov, S.A. and G.P. Georgiev. 1980. Non-random cleavage of SV40 DNA in the compact minichromosome and free in solution by micrococcal nuclease. Biochem. Biophys. Res. Commun. 92: 532-539.

Neigeborn, L. and M. Carlson. 1984. Genes affecting the regulation of SUC2 gene expression by glucose repression in Saccharomyces cerevisiae. Genetics 108: 845-858.

Neigeborn, L., K. Rubin, and M. Carlson. 1986. Suppressors of snf2 mutations restore invertase derepression and cause temperature-sensitive lethality in yeast. Genetics 112: 741753.

Nelson, R.G. and W.L. Fangman. 1979. Nucleosome organization of the yeast 2- $\mu \mathrm{m}$ DNA plasmid: A eukaryotic minichromosome. Proc. Natl. Acad. Sci. 76: 6515-6519.

Norris, D., B. Dunn, and M.A. Osley. 1988. The effect of histone gene deletions on chromatin structure in Saccharomyces cerevisiae. Science 242: 759-761.

Paro, R. 1990. Imprinting a determined state into the chromatin of Drosophila. Trends Genet. 6: 416-421.

Peterson, C.L. and I. Herskowitz. 1992. Characterization of the yeast $S W I 1, S W I 2$, and $S W I 3$ genes, which encode a global activator of transcription. Cell 68: 573-583.

Peterson, C.L., W. Kruger, and I. Herskowitz. 1991. A functional interaction between the C-terminal domain of RNA polymerase II and the negative regulator SIN1. Cell 64: 11351143.

Rose, M.D., F. Winston, and P. Hieter. 1990. Methods in yeast genetics: A laboratory course manual. Cold Spring Harbor Laboratory Press, Cold Spring Harbor, New York.

Sarokin, L. and M. Carlson. 1984. Upstream region required for regulated expression of the glucose-repressible SUC2 gene of Saccharomyces cerevisiae. Mol. Cell. Biol. 4: 2750-2757.

Sherman, F., G.R. Fink, and C.W. Lawrence. 1978. Methods in yeast genetics, rev. ed. Cold Spring Harbor Laboratory, Cold Spring Harbor, New York.

Som, T., K.A. Armstrong, F.C. Volkert, and J.R. Broach. 1988. Autoregulation of $2 \mu \mathrm{m}$ circle gene expression provides a model for maintenance of stable plasmid copy levels. Cell 52: 27-37.

Stern, M., R. Jensen, and I. Herskowitz. 1984. Five SWI genes are required for expression of the $\mathrm{HO}$ gene in yeast. J. Mol. Biol. 178: 853-868.

Swanson, M.S. and F. Winston. 1992. SPT4, SPT5, and SPT6 interactions: Effects on transcription and viability in Saccharomyces cerevisiae. Genetics 132: 325-336.

Swanson, M.S., M. Carlson, and F. Winston. 1990. SPT6, an essential gene that affects transcription in Saccharomyces cerevisiae, encodes a nuclear protein with an extremely acidic amino terminus. Mol. Cell. Biol. 10: 4935-4941.

Swanson, M.S., E.A. Malone, and F. Winston. 1991. SPT5, an essential gene important for normal transcription in Saccharomyces cerevisiae, encodes an acidic nuclear protein with a carboxy-terminal repeat. Mol. Cell. Biol. 11: 3009-3019.

Tamkun, J.W., R. Deuring, M.P. Scott, M. Kissinger, A.M. Pattatucci, T.C. Kaufman, and J.A. Kennison. 1992. brahma: A regulator of Drosophila homeotic genes structurally related to the yeast transcriptional activator SNF2/SWI2. Cell 68: 561-572.

Travers, A.A. 1992. The reprogramming of transcriptional competence. Cell 69: 573-575.

Tremethick, D., K. Zucker, and A. Worcel. 1990. The transcription complex of the 5 S RNA gene, but not transcription factor IIIA alone, prevents nucleosomal repression of transcription. f. Biol. Chem. 265: 5014-5023.

van Holde, K.E. 1988. Chromatin. Springer-Verlag, New York.

Winston, F. and M. Carlson. 1992. Yeast SNF/SWI transcriptional activators and the SPT/SIN chromatin connection. Trends Genet. 8: 387-391.

Winston, F., D.T. Chaleff, B. Valent, and G.R. Fink. 1984. Mutations affecting Ty-mediated expression of the HIS4 gene of Saccharomyces cerevisiae. Genetics 107: 179-197.

Workman, J.L. and R.G. Roeder. 1987. Binding of transcription factor TFIID to the major late promoter during in vitro nucleosome assembly potentiates subsequent initiation by RNA polymerase II. Cell 51: 613-622.

Workman, J.L., I.C.A. Taylor, and R.E. Kingston. 1991. Activation domains of stably bound GAL4 derivatives alleviate repression of promoters by nucleosomes. Cell 64: 533-544.

Wu, C. 1980. The $5^{\prime}$ ends of Drosophila heat shock genes in chromatin are hypersensitive to DNase I. Nature 286: 854 860 . 


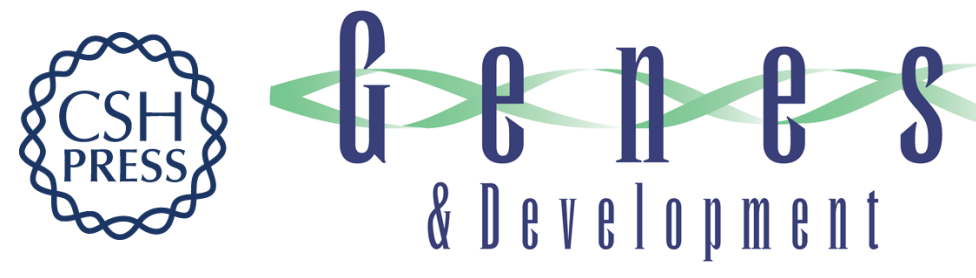

\section{Evidence that SNF2/SWI2 and SNF5 activate transcription in yeast by altering chromatin structure.}

J N Hirschhorn, S A Brown, C D Clark, et al.

Genes Dev. 1992, 6:

Access the most recent version at doi:10.1101/gad.6.12a.2288

References This article cites 62 articles, 33 of which can be accessed free at:

http://genesdev.cshlp.org/content/6/12a/2288.full.html\#ref-list-1

License

Email Alerting Receive free email alerts when new articles cite this article - sign up in the box at the top Service right corner of the article or click here.

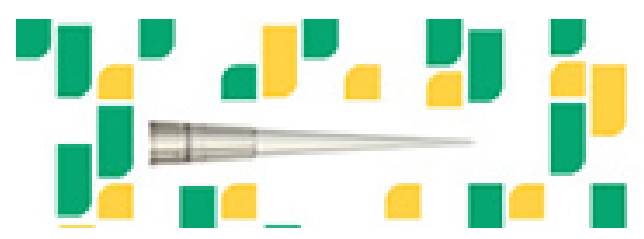

Focused on your science.

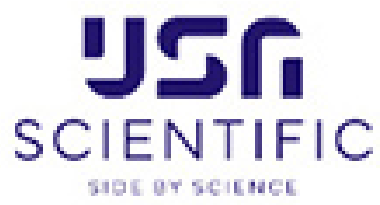

Copyright (c) Cold Spring Harbor Laboratory Press 